\title{
Superb Posner - but can we go further?
}

Frey, Bruno S

\begin{abstract}
Posner's (2010) analysis offers many exciting insights into the principal-agent problem, particularly with respect to the secret service. I argue that it would be useful to consider a broader model of human behaviour, which includes awards as extrinsic incentives beyond pay, as well as intrinsic motivation. A more comparative stance that goes beyond the United States would be a useful check of how general the results are. Scholars should not forget that while the US is the dominant economy today, there are 195 nations in the world that offer many fascinating institutional variations, which are useful to take into account.
\end{abstract}

DOI: https://doi.org/10.1017/S1744137409990166

Posted at the Zurich Open Repository and Archive, University of Zurich ZORA URL: https://doi.org/10.5167/uzh-44420

Journal Article

Published Version

Originally published at:

Frey, Bruno S (2010). Superb Posner - but can we go further? Journal of Institutional Economics, 6(01):65-69.

DOI: https://doi.org/10.1017/S1744137409990166 


\title{
Superb Posner - but can we go further?
}

\author{
BRUNO S. FREY* \\ University of Zurich, Zurich, Switzerland
}

\begin{abstract}
Posner`s (2010) analysis offers many exciting insights into the principal-agent problem, particularly with respect to the secret service. I argue that it would be useful to consider a broader model of human behaviour, which includes awards as extrinsic incentives beyond pay, as well as intrinsic motivation. A more comparative stance that goes beyond the United States would be a useful check of how general the results are. Scholars should not forget that while the US is the dominant economy today, there are 195 nations in the world that offer many fascinating institutional variations, which are useful to take into account.
\end{abstract}

\section{Posner`s performance}

Richard Posner is one of the most productive scholars in the social sciences. He is at the same time an able academic teacher, a prolific author of books and papers, an Appeals Court judge, and covers a very broad range of subjects. His paper 'From the new institutional economics to organization economics' demonstrates these wide-ranging abilities again. He is an impressive example that high quantity is well compatible with high quality of scholarly production.

Needless to say, I am a great admirer of Richard Posner. It is therefore a great challenge to me to make suggestions on how this paper can be further improved: where are its limitations, disregarded aspects, and what different avenues would be fruitful?

A great strength of his (2010) paper is that he does not linger on methodological issues, but applies his approach to real world issues. I will mainly focus on his most fascinating and insightful analysis of the secret service. Posner's earlier paper with Garicano (2005) is, in my view, one of the best recent papers in economics. But let me start with a general critique of how Posner enlarges standard economics by using psychological and sociological concepts such as 'culture', 'incalculated norms of workers', or 'high commitment' (Section 2). I then discuss incentive issues in the secret service (Section 3), and consider Posner's suggestions of how individuals should be compensated (Section 4).

*Email: bsfrey@iew.uzh.ch.

The author wishes to thank Margit Osterloh and Isabel Ellenberger. 


\section{Standard economics and psychological and sociological concepts}

Everyone who has read Richard Posner's work is well aware that he is one of the staunchest defenders of the standard economic model of human behaviour. This stance is clearly reflected in his (2010) paper. For example, he states that he does 'not like' the idea of 'bounded rationality' ( $\mathrm{p} . \mathrm{xx}$ ), and that 'the normal incentive' that individuals respond to is monetary income (p. $\mathrm{xx}$ ). Posner is not part of that group of social scientists who believe that progress can mainly (or even only) be made by introducing insights and concepts from psychology, sociology, or other social sciences. Rather, he endeavours to present the insights that can be gained by fully exploiting the standard theory based on a selfish homo oeconomicus.

But Richard Posner does not simply stick to his own programme. He actually uses many concepts clearly borrowed from psychology and sociology. Thus, for example, on page $\mathrm{xx}$, he refers to 'culture', 'common norms and understandings' and 'customs', on page $\mathrm{xx}$ to 'incalculating workers with professional norms' and to their 'high-commitment', and on pages $\mathrm{xx}$ and $\mathrm{xx}$ 'career satisfaction' plays a major role. He even takes these intrinsic motivations to mark the difference between new institutional economics, which disregards aspects 'such as highcommitment environment and other variables of organizational culture' (p. $\mathrm{xx})$ and the type of organizational economics he propounds. Such a position (provided it is factually true) would be no problem for a scholar relying on interdisciplinarity to gain insights. But it is a problem for Posner`s approach, seeking to explain reality with standard material preferences. In fact, he uses these psychological and sociological elements only when the purely selfish homo oeconomicus no longer fits, leading to '...perplexed organizational economists' (p. xx). These elements are introduced in an ad hoc way to make the standard economic model compatible with reality. As a result, Posner`s account reflects reality well, but no longer provides empirically testable insights. The appropriate procedure would be to start from a general and consistent model of human behaviour in which the psychological and sociological elements are an essential part. In particular, a consistent approach would acknowledge that an individual's motivation combines both extrinsic and intrinsic elements. In his discussion of corporate compensation (Section 4.1), Posner emphasises the importance of 'nonpecuniary rewards for work' (p. xx), but disregards that under well-identified empirical conditions extrinsic and intrinsic motivations interact, leading to crowding-in or crowding-out effects of intrinsic motivation (e.g. Frey, 1997; Bénabou and Tirole, 2003, 2006). It is thus in many situations impossible to simply add intrinsic to extrinsic motivations as Richard Posner seems to think.

\section{Incentives in the secret service}

As already stated above, the discussion of issues of centralization vs. decentralization, the independent sources of information needed, the turf wars 
within intelligence, and the diseconomies of scope provide most striking and brilliant insights. However, the analysis of the relevant incentives is lacking. In one respect, this is rather inconsistent. Posner starts the discussion by noting: 'Intelligence is ... a widely unpopular career in democratic society' (p. xx), which implies a concern for social standing. Afterwards he says: ' . . nonpecuniary income is a major element of the full income of an intelligence officer' ( $\mathrm{p} . \mathrm{xx}$ ) because he observes that ten times as many people apply for jobs with the secret service than there are jobs available. This is yet another case in which the intrinsic aspects of nonpecuniary income are added to the analysis in an ad boc way so as to suit what has been discovered empirically. The analysis of the secret service could be improved in two regards.

Firstly, the utility function of the people working in intelligence (as well as of all other people) should be formulated in a more differentiated way. In addition to what Richard Posner considers the 'normal incentive' (p.xx), another extrinsic incentive of considerable importance exists: awards in the form of orders, crosses, prizes, and titles (see Frey, 2006). It has been argued that they may partly compensate for lower income (Frey and Neckermann, 2008). Interestingly enough, awards are also of great relevance in for-profit enterprises. Indeed, American capitalist firms hand out a huge number of awards. It is important to see that awards differ from income. They are understood in a different way, namely as a form to offer honour and recognition to the recipients. Awards are given according to different principles, typically according to vague criteria and ex post. In the secret service, as part of the public sector they serve mainly to strengthen identification and commitment, i.e. exactly those factors stressed by Posner in his analysis.

Secondly, while Richard Posner sets the intelligence community against the FBI, a more comparative approach would yield even more insights. It would certainly be most fruitful to compare the US secret service to the one in the United Kingdom, or to any in other major democracy, as well as to those in non-democratic countries. It would be equally insightful to consider how secret services were handled in the past. Thus, for example, Hitler tried to solve the information problem, which has been identified so well by Posner, by giving the identical task to the commander of several different secret services. Such behaviour was thus not a sign of lacking adeptness, but of rational considerations (see Breton and Wintrobe, 1986).

\section{Determining compensation}

I perfectly agree with Richard Posner that '. . objective output measures of any kind are unlikely to be highly correlated with the desired output' (p. xx). I would be even more critical of any attempt to use 'quantitative criteria of judicial performance' (p. xx). If judges are paid according to 'performance', they will neglect all aspects not explicitly part of such a measure (this is the well-known 
multi-tasking problem), their intrinsic motivation will be crowded out (because the measure explicitly indicates that it is considered unnecessary), and they could skilfully 'game the system'.

Posner takes the compensation problem to be particularly serious in the judiciary, and he therefore suggests that compensation should be linked to the 'quality of its inputs' (p. xx), which means that any hiring process has to be undertaken carefully. But there is yet another possible criterion which Posner misses, namely 'process control' (see Osterloh et al., 2009), where employees are evaluated according to how well they conform to professional standards that govern how tasks are to be undertaken. The professional norms have to be incalculated (to refer again to one of Posner`s major themes) so that they become an intrinsic part of behaviour. This procedure has been the established method in which German bureaucracy functioned in the past. While certainly not without problems, it was able to be quite efficient in the sense of providing the services in a fair and non-corrupt way (see Weber, 1987).

\section{Conclusion}

Posner's paper is delightful and offers many new and exciting insights, particularly with respect to the secret service. However, I have argued that a broader model of human behaviour, including awards as extrinsic incentives beyond pay, as well as intrinsic motivation, would be useful to consider. Also, a more comparative stance beyond the United States would be a useful check of how general the results are. Scholars should not forget that while the US is the dominant economy today, the world composed of 195 nations offers many fascinating institutional variations, which would be useful to take into account.

\section{References}

Bénabou, R. and J. Tirole (2003), 'Intrinsic and extrinsic motivation', Review of Economic Studies, 70(3): 489-520.

Bénabou, R. and J. Tirole (2006), 'Incentives and prosocial behavior', American Economic Review, 96(5): 1652-1678.

Breton, A. and R. Wintrobe (1986), 'The bureaucracy of murder revisited', Journal of Political Economy, 94(5): 905-926.

Frey, B.S. (1997), Not Just For the Money, Cheltenham, UK: Edward Elgar.

Frey, B. S. (2006), 'Giving and receiving awards', Perspectives on Psychological Science, 1: 377-388.

Frey, B.S. and S. Neckermann (2008), 'Awards: a view from psychological economics', Journal of Psychology, 216(special issue): 198-208.

Garicano, L. and R. A. Posner (2005), 'Intelligence failures: an organizational economics perspective', Journal of Economic Perspectives, 19(4): 151-170. 
Osterloh, M., B. S. Frey, and F. Homberg (2009), 'Performance evaluation and pay for performance: does it really motivate public officials?', Working Paper, Institute for Empirical Research in Economics, University of Zurich.

Posner, Richard A. (2010), 'From the new institutional economics to organization economics: with applications to corporate governance, government agencies, and legal institutions', Journal of Institutional Economics, 6(1): 1-37.

Weber, M. (1987), Economy and Society, G. Roth and C. Wittich (eds.), Berkeley, CA: University of California Press. 